\section{Adapting Dialectical Behavior Therapy for an Adolescent with Neurofibromatosis 1: A Case Study}

\section{Abstract}

Background: There remains a need for interventions addressing psychopathology in individuals with cognitive and intellectual disability (ID). This case examines the application of Dialectical Behavior Therapy (DBT) in an adolescent with Neurofibromatosis 1 (NF1) and multiple psychiatric diagnoses. Treatment targeted suicidal ideation (SI), non-suicidal self-injury (NSSI) and impulsivity, while addressing cognitive limitations.

Case background: The patient met criteria for Major Depressive Disorder, Borderline Personality Disorder, and Attention-Deficit Hyperactivity Disorder. She had a two-year history of NSSI behavior and two prior psychiatric inpatient hospitalizations. At baseline, she presented with deficits in interpersonal relationships, emotional reactivity and maternal conflict.

Course of treatment: The treatment plan followed the DBT-A protocol, with modifications made to address the patient's cognitive limitations. Adaptions included greater reliance on behavioral and visual learning strategies, increased maternal involvement, regular collaboration with the multidisciplinary care team, and shortened sessions to fit the patient's attentional capability.

Treatment outcomes: The patient showed a reduction in NSSI, SI, and did not require further psychiatric hospitalization. Patient and mother evidenced increased skill use. Additional outcomes are discussed.

Conclusions: These adaptations offer a preliminary account of DBT-A for an adolescent with cognitive impairments. Important factors for successful modification included a multidisciplinary treatment approach, adapted and targeted teaching methods, and significant reliance on behavioral principles. This case study suggests that DBT is a promising treatment for individuals with ID and cognitive impairments and should be further tested within this population.

Keywords: Adolescent behavior; Self-injurious behavior; Adolescent psychology; Borderline personality disorder
Joslyn Kenowitz ${ }^{1 *}$, Courtney Santucci ${ }^{2}$, Michelle Lupkin², Lesley Michael ${ }^{3}$ and Sandra Pimentel ${ }^{2}$

1 Nemours/A.I. duPont Hospital for Children, Wilmington, Delaware, United States

2 Albert Einstein College of Medicine, Montefiore Medical Center, Bronx, NY, United States

3 Lenox Hill Hospital, Northwell Health, New York, United States

*Corresponding author:

Joslyn Kenowitz, Ph.D

” Joslyn.kenowitz@gmail.com

Division of Behavioral Health, Nemours/A.I. duPont Hospital for Children, 1801 Rockland Road, Wilmington, DE 19803, Delaware, United States.

Tel: 203-442-3183

Citation: Kenowitz J, Santucci C, Lupkin M, Michael L, Pimentel S (2018) Adapting Dialectical Behavior Therapy for an Adolescent with Neurofibromatosis 1: A Case Study. J Child Dev Disord. Vol.4 No.4:14

Received: August 31, 2018; Accepted: October 29, 2018; Published: November 06, 2018

\section{Introduction}

Dialectical Behavior Therapy (DBT) was first introduced as a cognitive behavioral therapy targeting chronic suicidality among adults with borderline personality disorder (BPD) [1-3]. DBT was later adapted by Miller and colleagues [4] to address the severe pathology of adolescents presenting with multiple problems, including suicidal ideation (SI) and non-suicidal self-injury (NSSI).
Early research in the effectiveness of this treatment modality with this specific population gave rise to empirical evidence to support the utility of this treatment among adolescents presenting with multiple problems [5-7]. Maintaining its core principles and fundamental structure, DBT for adolescents (DBT-A) required some modification and adaptation to suit the unique needs of the youth presenting with emotion dysregulation. 
Similar to the treatment designed for adults [2], DBT-A incorporates four modes of treatment into therapy- weekly individual therapy, weekly skills group training, phone skills coaching, and weekly consultation team meetings. Individual sessions allow for a focused approach to addressing target behaviors in order of highest priority, and make use of weekly diary cards, which record the individual's emotions, target urges and behaviors, and their use of skills. Complementing these individual sessions, patients and at least one parent or guardian are required to participate in weekly, multi-family skills groups. The inclusion of family members has been found to have the following benefits: generalization of skills, opportunities for role play of skills in-vivo, reduction of disruptive behaviors, interfamily support, the reinforcement of coaching and skills use [1], and greater probability that the adolescent will complete group [8]. The multi-family group focuses on the acquisition of new skills in each of the five modules - mindfulness, distress tolerance, interpersonal effectiveness, emotion regulation, and middle path skills [1].

In an effort to support the generalization of skills, to provide emergency interventions, to positively attend to and reinforce "good news," and to promote a healthy therapeutic relationship, phone skills coaching is also utilized for both the adolescents and their parents. In DBT-A, adolescents are allowed to contact their individual therapist, while their parent is allowed to contact their skills group leader at any time they are in need of individualized skills coaching [1].

Finally, in order to effectively maintain adherence to the format of DBT, weekly consultation team meetings are held for therapists. Team meetings allow therapists to consult with members of the DBT team and to provide "therapy to the therapists" who are treating patients with multiple problems [1].

In addition to the treatment of adolescents with BPD, DBT has been adapted, applied, and examined in a variety of ages and psychological disorders. For example, DBT skills training in adolescents with oppositional defiant disorder showed improvement in behaviors as well as internalizing symptoms [9]. The use of DBT in the treatment of adolescents with eating disorders also showed significant improvements in specific symptoms of anorexia and bulimia, as well as overall psychopathology [10,11]. Goldstein and colleagues [12] also found that using DBT to treat adolescents with bipolar disorder significantly decreased suicidality, NSSI, depressive symptoms, and emotion dysregulation. Additionally, randomized control trials have shown DBT to be successful in improving coping skills and reducing suicidal ideation and depressive symptoms in preadolescent children [13] as well as improving functioning in adults with post-traumatic stress disorder, substance use disorders, and eating disorders [14]. Finally, recent work by Hashim and colleagues [15] also showed DBT to be a promising intervention for improving treatment adherence in adolescents with chronic kidney disease.

Despite the successful adaptations of DBT, there remains a need for inclusive, comprehensive therapies addressing psychopathology in individuals with intellectual disability (ID) and

cognitive impairment. While there is no specific DBT protocol for individuals with these conditions, mindfulness based therapies and case studies assessing the use of features related to DBT in an ID population have shown promising results. In their review of mindfulness based approaches (i.e., ACT and DBT) in individuals with ID, Harper and colleagues [16] found mindfulness based therapies to reduce aggression, levels of stress, depression, and anxiety, as well as the frequency of NSSI. Similarly, Lew and colleagues [17] found a reduction in NSSI in the majority of a small sample of women with intellectual disability. Finally, a longitudinal study showed the gradual reduction of challenging behaviors in adults with ID who were treated with modified DBT [18]. Modifications included adapted diary cards (e.g. reducing text and including pictures). Additionally, strategies were modified to suit the individual's reading, writing, and cognitive level [18]. Despite these encouraging results, the literature on DBT in individuals with ID and presenting psychopathologies is limited and does not address an adolescent population.

Thus, this case study aims to add to the limited literature by offering a preliminary account of DBT-A for an adolescent presenting with severe psychopathology, Neurofibromatosis 1 (NF1) and related cognitive deficits. NF1 is a genetic disorder resulting in concomitant biological, psychosocial, development and cognitive challenges, due to the growth of benign and malignant tumors in the nervous system. The most reported complication of NF1 is neurocognitive impairments, with most individuals presenting in the low average IQ range. Learning and language disorders, along with visuospatial, visuomotor, and motor coordination deficits, occur with $30-60 \%$ of children with NF1 [19]. Additionally, individuals with NF1 have a higher rate of behavioral difficulties, psychosocial challenges, Autism Spectrum Disorders, and Attention Deficit/Hyperactivity Disorder [20]. Due to the prevalence of cognitive and psychosocial challenges, it is recommended that individuals with NF1 receive comprehensive multidisciplinary care that includes routine neuropsychological evaluation, educational intervention, and consultation with mental health professionals to address behavioral and social difficulties as they arise [19].

\section{Case Background and Conceptualization}

"Rachel" a 17-year-old, biracial female, was referred to an urban, hospital-based outpatient clinic after two inpatient hospitalizations for SI. At the time of intake, she endorsed a twoyear history of NSSI occurring three to four times per week. Rachel was diagnosed with NF1 at the age of 2, resulting in multiple surgeries and long-term cognitive impairments. Her academic functioning was reported to be at a $6^{\text {th }}$ grade learning level with her full scale intelligence quotient falling in the Borderline range. Rachel was a senior in high school at a special academic school that provided education and life skills to individuals with cognitive disabilities. Additionally, Rachel was diagnosed with ADHD in elementary school, which had since been successfully managed with medication. After clinical assessment with the Kiddie Schedule for Affective Disorders and Schizophrenia (K-SADS) 
and the Structured Interview for DSM-IV Personality (SIDP-IV), Rachel was diagnosed with Major Depressive Disorder (MDD) and Borderline Personality Disorder (BPD). While personality disorders are thought to be disorders of adulthood, there is a significant body of research to support the validity of a diagnosis of BPD in adolescence. Longitudinal and large-scale community research indicates that the presence of symptoms of personality disorders in adolescents reliably predicts personality disorders in adulthood. Furthermore, research suggests that the onset of symptoms typically begins at puberty, however, patients often do not present for treatment until older age, skewing the age of diagnosis to 18 [21]. As with other emerging youth diagnoses, early assessment, diagnosis, and appropriate intervention for BPD has the potential to reduce impairment and improve functioning [22].

Using the DBT biosocial framework, Rachel's diagnoses are best conceptualized as the breakdown of her emotion regulation system, resulting from the interaction of her illness and environmental factors. Various aspects of Rachel's chronic illness created a barrier to learning how to regulate her emotions, expressing and communicating emotions, and understanding the experiences of others. Rachel's medical condition required a significant amount of her mother's time and resources, limiting her capacity to tolerate the additional stressors of a developing adolescent. Rachel's mother, who presented with her own elevated stress, inadvertently invalidated many of Rachel's emotional experiences. This contributed to further emotion dysregulation. Due to lack of adaptive coping skills, Rachel attempted to regulate her emotions by engaging in NSSI and inappropriate attention seeking behaviors.

\section{Course of Treatment and Adaptations}

Consistent with the framework of DBT-A, Rachel was enrolled in weekly individual therapy, including phone skills coaching with her individual therapist, and weekly skills group training, led by a DBT-A trained psychologists and psychology intern. All members of her treatment team also met for weekly consultation team meetings. Although the treatment plan followed DBT-A protocol [1], some modifications were necessary given Rachel's cognitive limitations (Table 1). After explanation of the treatment hierarchy, Rachel was asked to identify goals for treatment, which most closely tied to emotional instability, interpersonal conflict, and family dilemmas. In the subsequent sessions, Rachel and the therapist worked on rating and anchoring Rachel's emotions so they could be more precisely reported on her diary card. Rachel was initially presented with a diary card that was used in DBT for younger children [13], as opposed to a diary card for adolescents. Suicidal ideation and NSSI urges were still tracked, however the amount of information collected was reduced and simplified as compared to the typical DBT-A diary card. Rachel collaborated with the therapist to make the diary card more visually appealing and create her own 0-4 scale to capture and describe the range and intensity of emotions throughout the day (Figure 1). The diary card helped Rachel identify the link between triggering situations, emotions, and SI/NSSI behaviors, which were visually displayed using simplified chain analyses.
Table 1 Summary of Adaptations and Behavioral Strategies.

\begin{tabular}{|c|c|c|}
\hline Limitation & Adaptation & Example \\
\hline $\begin{array}{l}\text { Difficulty with } \\
\text { orally presented } \\
\text { information }\end{array}$ & $\begin{array}{l}\text { - Visual presentation of } \\
\text { information }\end{array}$ & $\begin{array}{l}\text { - Use of a white board } \\
\text { to write in session } \\
\text { - Use of pictures }\end{array}$ \\
\hline $\begin{array}{c}\text { Intellectual } \\
\text { disability }\end{array}$ & $\begin{array}{l}\text { - Modified diary card } \\
\text { Simplified chain } \\
\text { analysis }\end{array}$ & $\begin{array}{l}\text { - } \begin{array}{l}\text { Simpler language on } \\
\text { diary card }\end{array} \\
\text { Fewer targets on } \\
\text { chain analysis } \\
\text { Multiple paging } \\
\text { practice trials in } \\
\text { therapist's office }\end{array}$ \\
\hline Attention span & $\begin{array}{ll}\text { - } & \text { Time limited } \\
\text { discussions } \\
\text { - } & \text { Shortened sessions }\end{array}$ & $\begin{array}{l}\text { - Using stop watch to } \\
\text { time discussions } \\
\text { - Ending session after } \\
35 \text { minutes }\end{array}$ \\
\hline Adaptive skills & $\begin{array}{l}\text { Greater support from } \\
\text { mother and school } \\
\text { personnel }\end{array}$ & $\begin{array}{l}\text { - Allowance of parent } \\
\text { to page individual } \\
\text { therapist } \\
\text { - Role of parent as } \\
\text { coach } \\
\text { - Weekly consultation } \\
\text { with school guidance } \\
\text { counselor }\end{array}$ \\
\hline
\end{tabular}

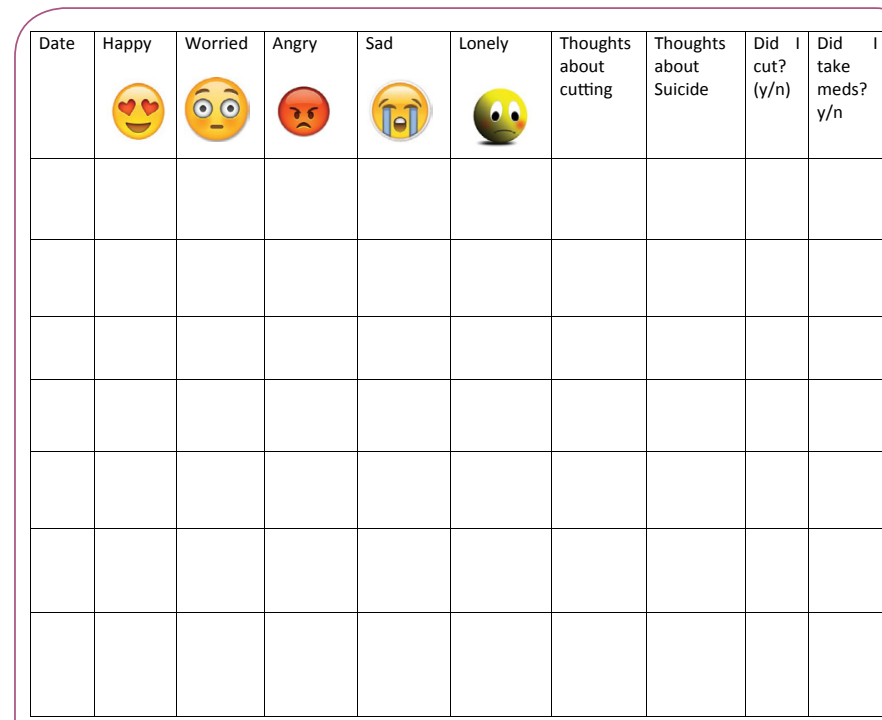

Emotion ratings

$0=$ Not at all

$1=$ A little bit

$2=$ Somewhat

$3=$ A lot (really)

$4=$ Extremely

Figure 1 Modified diary card.
Coping skills

Reading

Journal

Photography

seeing counselor

mindfulness

Paging therapist: $x x x-x x-x x x x$
DBT-A also relies on the use of paging an individual therapist to provide in-the -moment skills coaching when an adolescent is feeling dysregulated and is having urges for NSSI. Despite practice paging in session, Rachel had difficulty paging her 
therapist outside of session early in treatment. Rachel expressed resistance to paging and often reported that she forgot to page when feeling emotionally dysregulated. In order to shape Rachel's behavior, the therapist instituted a behavior plan whereby Rachel was to page the therapist every day for one week and receive a reward at the upcoming session for following through with the assignment.

As treatment progressed, it was clear that Rachel's mother needed to play a larger role in scaffolding the therapy. Rachel's mother was asked to act as a coach for Rachel outside of the clinic; when she noticed Rachel becoming emotionally dysregulated, or if Rachel made a comment about hurting herself, Rachel's mother coached Rachel to use a skill or page her therapist. Finally, given the importance of Rachel's mother in her care, Rachel's mother was allowed to page Rachel's individual therapist herself for coaching, rather than skills group leaders, as would typically occur in DBT-A.

The structure of individual sessions was also modified to fit Rachel's unique needs.

Each individual session included a review of skills from the prior group as a means of reinforcing new skills. Given Rachel's attentional needs, the therapist and Rachel began setting time limits for discussion, in which Rachel would receive a reward if she was fully engaged in shorter on-task conversations.

Finally, in addition to weekly consultation with the DBT team, the therapist had regular contact with other members of her treatment team. Rachel's guidance counselor was provided with handouts about each skill learned during the week, to further reinforce the use of skills outside the clinic. Information gathered from her neuropsychologist and neuropsychological evaluation guided the use of specific intervention strategies, including presenting information visually rather than verbally. Furthermore, her therapist, psychiatrist, and neurologist frequently consulted one another regarding progress in therapy, psychotropic medications, and progression of her disease.

\section{Psychopharmacological treatment}

At the time of intake, Rachel was prescribed dextroamphetamine and amphetamine extended-release $30 \mathrm{mg}$ daily for ADHD. Rachel had been compliant with this stimulant treatment for inattention and impulsivity since elementary school. Prior to admission into the clinic, the stimulant was managed and prescribed by her neurologist every three months. Rachel was reportedly benefitting from this dose for many years without side effects. Her neurology team also communicated that there were no contraindications for its use in this case, despite her NF1 diagnosis and its associated manifestations and impairments. Rachel was also started on a selective serotonin reuptake inhibitor (SSRI), fluoxetine $20 \mathrm{mg}$ daily, during her first inpatient hospitalization when she exhibited at risk behavior (NSSI and SI) in the context of interpersonal relationships. Rachel was then readmitted immediately following this 10-day hospitalization after disclosing that she did not feel safe returning home. During the month-long second hospitalization, fluoxetine was maintained at $20 \mathrm{mg}$.
After Rachel's admission intake and completion of assessments and self-report scales in the outpatient psychiatry clinic, the team felt her irritability and self-reported depression could be better targeted by titrating her current SSRI. Rachel's neurology team was again consulted to ensure that there were no potential contraindications for increasing her medication within the context of her NF1. Titration occurred simultaneously with individual and group DBT sessions. Rachel was titrated to fluoxetine 40 mg with an improvement in mood and irritability and without reported side effects. Rachel's stimulant treatment for ADHD was also transferred to psychiatry as Rachel was attending monthly psychiatry appointments in the clinic and both her neurology and psychiatry teams felt this provided a more streamlined approach to medication management.

\section{Treatment Outcomes}

After initiation of treatment, Rachel showed a reduction in NSSI and $\mathrm{SI}$ per diary card report. She did not require psychiatric hospitalization over the course of 8 months of treatment. Rachel reported two instances of NSSI behaviors at the beginning of treatment, a significant decrease from the time of intake when she reported NSSI behaviors 3-4 times per week. Additionally, Rachel reported fewer and less severe depressive symptoms at the end of treatment. She endorsed more positive emotions on her diary card and through verbal report to therapist in session. Rachel continued to report some difficulty with interpersonal relationships, an area of concern that was prevalent for her well before enrollment in DBT and initiation of medication. For Rachel, difficulty interpersonal relationships is likely best explained by her underlying psychopathology, as this is a core feature of BPD. Conflict in interpersonal relationships was likely maintained through the breakdown of Rachel's emotion regulation system, resulting from the interaction of biological and environmental factors; Rachel's emotional vulnerability, stemming from biological and medical factors, interacted with an invalidating environment, which was inconsistently responsive to or negated her emotional experience, leading to extreme emotion dysregulation. However, Rachel actively practiced mindfulness skills and completed chain analyses in session, resulting in improved understanding of the link between interpersonal conflict, negative mood, and $\mathrm{SI} /$ NSSI urges and behaviors. This increased awareness resulted in the use of skills, rather than impulsive behaviors, to regulate emotions. Rachel's use of the pager increased after behavioral rehearsal and contingent reinforcement of paging. She reported feeling more comfortable and confident paging therapist after practicing. Rachel's use of paging also increased after her mother began to act as a coach, prompting Rachel to page the therapist when dysregulated or experiencing SI or NSSI urges.

Rachel gained a number of skills through participation in skills group and rehearsal of skills in individual therapy. When feeling angry or upset, she reported often using distress tolerance skills. She also exhibited successful use of interpersonal effectiveness skills which helped her communicate her feelings and needs in a clear, non-judgmental way. The use of skills also significantly changed the relationship between Rachel and her mother. Rachel's mother used distress tolerance stills to regulate her 
own emotions; by doing so, she was able to appropriately model and coach Rachel to page her therapist when Rachel became dysregulated or had urges to engage in NSSI. Rachel's mother reported particular use of middle path skills, which aim to effectively manage the unique adolescent-family dilemmas [1]. Within this skill set, Rachel's mother demonstrated a significant improvement in validation and behavioral skills.

\section{Conclusions and Implications for Practice}

This case report offers a preliminary account of DBT-A for an adolescent with cognitive impairment and highlights factors needed for a successful modification of DBT-A. Given the pervasiveness of the impairments, it is essential for the treatment approach to be multidisciplinary in nature. In order to adapt DBT-A for Rachel, consultation was extended outside of the DBT treatment team to Neurology, Psychiatry, Psychology, and Educational professionals. Providing education and DBT materials to the extended treatment team allowed for open dialogue, consistency, and consultation among each provider resulting in individualized therapy while keeping the framework of DBT-A.

Secondly, this adaptation of DBT-A revealed the significance of behavioral techniques for treating an individual with cognitive impairments. While DBT-A does use behavioral strategies and includes the teaching of behavioral principles in multi-family skills group, we found that behavioral techniques were the primary source of change in behavior for Rachel and her mother. Cognitive strategies were tested, however it was clear that this approach was too complicated for Rachel, as she was unable to synthesize this information and understand its purpose and use. Rachel was, however, able to perform simpler cognitive tasks such as emotion identification and recognizing the connection between feelings and behaviors. This is consistent with prior research applying

\section{References}

1. Miller AL, Rathus JH, Linehan MM (2007) Dialectical behavior therapy with suicidal adolescents. New York, NY: Guilford Press; p: 346.

2. Linehan MM (1993) Cognitive-behavioral treatment of borderline personality disorder. New York, NY: Guilford Press; p: 558.

3. Linehan MM (1993) Skills training manual for treating borderline personality disorder. New York, NY: Guildford Press; p: 180.

4. Miller AL, Rathus JH, Linehan MM, Wetzler S, Leigh E (1997) Dialectical behavior therapy adapted for suicidal adolescents. J Pract Psychiatr Behav Health 3: 78-86.

5. Katz I, Cox B, Gunarekara S, Miller A (2004) Feasibility for dialectical behavior therapy for suicidal adolescents inpatients. J Am Acad Child Adolesc Psychiatry 43: 276-282.

6. Rathus JH, Miller AL (2002) Dialectical behavior therapy adapted for suicidal adolescents. Suicide Life Threat Behav 32: 146-157.

7. Woodberry KA, Popenoe EJ (2008) Implementing dialectical
DBT to individuals with ID [18] as well as research supporting the effectiveness of applied behavior analysis rather than cognitive behavioral therapy for individuals with impairments [23].

Finally, this case highlights the need for assessment tools validated in a sample of individuals with cognitive or intellectual disability. Typically, adolescents in our clinic complete multiple self-report assessments at time of intake, midway through treatment, and at completion of multi-family skills group. These measures assess suicidal ideation, reasons for living, problem areas associated with BPD, and difficulties in emotion regulation. However, these measures are not validated in a population with cognitive limitations. Rachel had significant difficulty with word comprehension on these measures, and therefore was unable to accurately interpret and complete these questions. Thus, we were unable to obtain usable data that quantitatively captured treatment targets and progress.

While this case study focused on treating an individual with NF1, the adaptations noted here are applicable to other complex medical conditions with similar cognitive and intellectual challenges. Individuals with intellectual and cognitive impairment tend to have fewer psychological resources (e.g. coping skills) and are at risk for facing stressors associated with the development of psychopathology [24-26]. Despite this finding, there are limited approaches available to treating high-risk individuals with intellectual and cognitive disabilities. DBT provides a multimodal approach that permits for flexibility within the treatment targets. It emphasizes therapeutic techniques that skills that directly address the deficits of each unique individual being treated. This case study suggests that DBT is a promising treatment for individuals similar to Rachel and should be further tested within this population $[27,28]$.

\section{Conflict of Interest}

There are no conflicts of interest to declare.

behavior therapy with adolescents and their families in a community outpatient clinic. Cogn Behav Pract 15: 277-286.

8. Germán M, Corcoran V, Wheeler L, DeMairo J, Peros O, et al. (2018) Risk factors for early and late dropout from dialectical behavior therapy for suicidal adolescents. Behav Ther 41: 69-80.

9. Nelson-Gray R, Keane SP, Hurst RM, Mitchell JT, Warburton JB, et al. (2006) A modified DBT skills training program for oppositional defiant adolescents: romising preliminary findings. Behav Res Ther 44: 1811-1820.

10. Safer DL, Lock J, Couturier JL (2007) Dialectical behavior therapy modified for adolescent binge eating disorder: a case report. Cogn Behav Pract 14: 157-167.

11. Salbach-Andrae H, Bohnekamp I, Pfeiffer E, Lehmkuhl U, Miller AL (2008) Dialectical behavior therapy of anorexia and bulimia nervosa among adolescents: A case series. Cogn Behav Pract 15: 415-425.

12. Goldstein TR, Axelson DA, Birmaher B, Brent DA (2007) Dialectical behavior therapy for adolescents with bipolar disorder: A 1-year open trial. J Am Acad Child Adolesc Psychiatry 46: 820. 
13. Perepletchikova F, Axelrod SR, Kaufman J, Rounsaville BJ, DouglasPalumberi $\mathrm{H}$, et al. (2011) Adapting dialectical behaviour therapy for children: towards a new research agenda for paediatric suicidal and non-suicidal self-injurious behaviours. Child Adolesc Ment Health 16: $116-121$.

14. Harned MS, Korslund KE, Foa EB, Linehan MM (2012) Treating PTSD in suicidal and self-injuring women with borderline personality disorder: Development and preliminary evaluation of a Dialectical Behavior Therapy Prolonged Exposure protocol. Behav Res Ther 50 : 381-386.

15. Hashim BL, Vadnais M, Miller AL (2013) Improving adherence in adolescent chronic kidney disease: a dialectical behavior therapy (DBT) feasibility trial. Clin Pract Pediatr Psychol 1: 369.

16. Harper SK, Webb TL, Rayner K (2013) The effectiveness of mindfulness-based interventions for supporting people with intellectual disabilities: a narrative review. Behav Modif 37: 431-453.

17. Lew M, Matta C, Tripp-Tebo C, Watts D (2006) Dialectical behavior therapy (DBT) for individuals with intellectual disabilities: a program description. Ment Health Asp Dev Disabil 9: 1.

18. Brown JF, Brown MZ, Dibiasio P (2013) Treating individuals with intellectual disabilities and challenging behaviors with adapted dialectical behavior therapy. J Ment Health Res Intellect Disabil 6: 280-303.

19. Ferner R, Huson S, Thomas N, Moss C, Willshaw H, et al. (2007) Guidelines for the diagnosis and management of individuals with neurofibromatosis 1. J Med Genet 44: 81-88.
20. Williams V, Lucas J, Babcock M, Gutmann D, Korf B, et al. (2009) Neurofibromatosis type 1 revisited. Pediatrics 123: 124-133.

21. Paris J (2013) Diagnosing borderline personality disorder in adolescence. Ann Am Soc Adolesc Psychiatr 29: 237.

22. Courtney-Seidler EA, Klein D, Miller AL (2013) Borderline personality disorder in adolescents. Clin Psychol: Sci Pract 20: 425-444.

23. Sturmey P (2012) Treatment of psychopathology in people with intellectual and other disabilities. Can J Psychiatry 57: 593-600.

24. Taylor JL, Lindsay WR, Willner P (2008) CBT for people with intellectual disabilities: emerging evidence, cognitive ability and IQ effects. Behav Cogn Psychother 36: 723-733.

25. Cohen J, Levy H, Sloan J, Dariotis J, Biesecker B (2015) Depression among adults with neurofibromatosis type 1: prevalence and impact on quality of life. Clin Genet 88: 425-430.

26. Ejerskov C, Lasgaard M, Østergaard JR (2015) Teenagers and young adults with neurofibromatosis type 1 are more likely to experience loneliness than siblings without the illness. Acta Paediatr 104: 604-609.

27. Graf A, Landolt MA, Mori AC, Boltshauser E (2006) Quality of life and psychological adjustment in children and adolescents with neurofibromatosis type 1. J Pediatr 148: 348-353.

28. Martin S, Wolters P, Baldwin A, Gillespie A, Dombi E, et al. (2012) Social-emotional functioning of children and adolescents with neurofibromatosis type 1 and plexiform neurofibromas: relationships with cognitive, disease, and environmental variables. J Pediatr Psychol 37: 713-724. 